\title{
Perceived quality of life (QOLIE-31-P), depression (NDDI-E), anxiety (GAD-7), and insomnia in patients with epilepsy attended at a refractory epilepsy unit in real-life clinical practice
}

\author{
Alicia Gonzalez-Martinez ${ }^{1,2}$ (D) Álvaro Planchuelo-Gómez ${ }^{3}$ (D) Alba Vieira Campos ${ }^{4}$. Francisco Martínez-Dubarbie ${ }^{5}$. \\ José Vivancos ${ }^{1} \cdot$ María De Toledo-Heras ${ }^{4}$
}

Received: 8 April 2021 / Accepted: 6 September 2021 / Published online: 15 September 2021

(c) Fondazione Società Italiana di Neurologia 2021

\begin{abstract}
Objectives This study aims to evaluate the relationship between psychiatric comorbidity (anxiety and depression), somnolence, and quality of life, using validated scales in patients with epilepsy in real-life clinical practice and clinical and demographic variables.

Methods A cross-sectional observational study was conducted. Self-administered scales of anxiety disorders (GAD-7), depression (NDDI-E), somnolence (Epworth Sleepiness Scale (ESS)), and quality of life (QOLIE-31-P) in patients with epilepsy treated in the refractory epilepsy unit of a tertiary hospital were employed.

Results Eighty-four patients, $44.3 \pm 17.4$ years, $48.2 \%$ women, epilepsy duration $21.5 \pm 15.9$ years, and number of antiepileptic drugs $1.9 \pm 1.2$ were included. Severe anxiety was present in $14.3 \%$, depression in $20.2 \%$, and somnolence in $14.3 \%$ of patients. QOLIE-31-P score was 62.0 \pm 19.2. Depression and focal epilepsy $(O R=4.5[1.3,20.7], p=0.029)$, as well as anxiety and temporal lobe epilepsy $(O R=4.3[1.0,18.1], p=0.044)$, were associated. Moreover, relationships between worse quality of life and higher scores from NDDI-E $(\beta=-1.42$, adjusted $p=0.006)$ and GAD-7 $(\beta=-1.21$, adjusted $p=0.006)$, especially in drug-resistant epilepsy $(\beta=-8.08$, adjusted $p=0.045)$ and female sex $(\beta=-7.83$, adjusted $p=0.034)$, were identified. Statistically significant negative associations were observed between problems to fall asleep and overall quality of life score $(\beta=-11.64$, adjusted $p=0.022)$, sleep disturbance and energy $(\beta=-14.78$, adjusted $p=0.027)$, and mood $(\beta=12.40$, adjusted $p=0.027)$ scores.

Conclusions The multidimensional evaluation revealed that higher levels of anxiety and depression are associated with worse quality of life in real clinical practice in patients with epilepsy, especially in females and drug-resistant epilepsy. In addition, sleep disturbances are associated with particular aspects of the quality of life. Further studies with longitudinal follow-up would be useful to adequately manage these comorbidities in patients with epilepsy.
\end{abstract}

Keywords Quality of life · Drug-resistant epilepsy · Anxiety · Depression · Sleep disturbances · QOLIE-31-P

Alicia Gonzalez-Martinez

alicia.gonzalez.martinez@live.com

1 Neurology Department, Hospital Universitario de La Princesa \& Instituto de Investigación Sanitaria La Princesa, Madrid, Spain

2 Hospital Universitario de la Princesa, Calle Diego de León, 62, 28006 Madrid, Spain

3 Imaging Processing Laboratory, Universidad de Valladolid, Valladolid, Spain

4 Epilepsy Unit, Neurology Department, Hospital Universitario de La Princesa \& Instituto de Investigación Sanitaria La Princesa, Madrid, Spain

5 Neurology Department, Hospital Marqués de Valdecilla, Santander, Spain

\section{Introduction}

Epilepsy is a frequent neurological disorder with a prevalence of approximately 6 per 1000 people [1]. In the past few years, the measurement of the quality of life (QoL) of patients with epilepsy has become an important practice [2]. The World Health Organization (WHO) defines QoL as a broad-ranging concept affected in a complex way by a person's physical health, psychological state, level of independence, social relationships, personal beliefs, and their relationship to salient features of their environment [3]. Several factors, such as seizure frequency, depression, 
anxiety, and sleep disturbances, have been associated with poor QoL in patients with epilepsy [4-6].

Psychiatric comorbidities, including mood and anxiety, are common in epilepsy, often occurring at rates two- to threefold or higher than in the general population without epilepsy [5]. At least $20 \%$ of patients with epilepsy suffer from depression [7], while its prevalence may reach $60 \%$ in patients with temporal lobe epilepsy [8]. Anxiety, often without concomitant depression, is also highly prevalent in patients with epilepsy and independently associated with focal/unknown and mesial temporal sclerosis epilepsy [9].

Regarding sleep disturbances, it has been demonstrated that sleep plays an intricate role in the disease process of epilepsy [10]. Poor sleep quality is associated with more frequent seizures [11]. In a meta-analysis, it has been reported that more than $10 \%$ of adults with epilepsy suffer sleep apnea-hypopnea syndrome (SAHS) [12]. Moreover, sleep comorbidity contributes to a worse QoL in patients with epilepsy [4].

Given the increasing body of evidence of the importance of psychiatric and sleep comorbidities in patients with epilepsy, the main objective of our study was the assessment of all the above-mentioned wide range of variables explored including QoL in relation to symptoms of anxiety and depression and sleep disorders, as well as clinical variables, in patients with epilepsy using selfadministered surveys in real clinical practice.

\section{Material and methods}

\section{Participants}

Patients with epilepsy were recruited from the epilepsy unit from the Hospital Universitario de La Princesa (Madrid, Spain), a tertiary hospital and national reference center for the treatment of refractory epilepsy. The patients were recruited consecutively between December 2019 and March 2020, before the beginning of the lockdown related to the COVID-19 pandemic in Spain.

Inclusion criteria were patients with a diagnosis of epilepsy for more than a year who were under antiepileptic drugs (AED). Exclusion criteria included cognitive disability that makes it difficult to adequately complete the survey.

During the in-person visit to the epilepsy unit, the patients were invited to take part in the study. A written informed consent was signed by the patients who accepted to participate, and self-administered surveys for each scale were provided to them. The research was approved by the ethics committee of the Hospital Universitario de La Princesa.

\section{Variables of study}

The main demographic and clinical variables were sex, age, total time with epilepsy, seizure frequency, occurrence of nonepileptic paroxysmal events, and number of AED. Based on the International League Against Epilepsy guidelines, drug-resistant epilepsy was defined as lack of success to provide trials of two or more tolerated, suitably selected and employed AED schedules to achieve sustained seizure freedom [13] according to the information available on the neurological medical records.

QoL was assessed using the Spanish version of the Patient-Weighted Quality of Life in Epilepsy inventory (QOLIE-31-P) questionnaire [14]. The QOLIE-31-P consists of 38 self-reported items and is divided in eight parts which evaluate energy (five items), mood (six items), daily activities (six items), cognition (seven items), medication effects (four items), seizure worry (six items), overall QoL (three items), and health state (one item). The first and the last items are scored with $0-10$ and $0-100$ scales, respectively, from the worst to the best situation. The remaining 36 items are scored using a Likert rating scale from 1 to $3,4,5$, or 6 . The raw scores from each subscale are reorganized to have scores from 0 to 100 . The final score is obtained from the subscale scores and is rescaled from 0 to 100, with higher scores associated with better QoL.

Symptoms of anxiety were analyzed using the 7-item generalized anxiety disorder (GAD-7) scale [15]. We provided the Spanish validated version [16]. These scores were considered according to the experiences from the last 15 days. In the Spanish version, a cut-off value of 10 was related to adequate values of sensitivity and specificity. Previous diagnosis of anxiety was obtained from the medical history.

The Neurological Disorders Depression Inventory for Epilepsy (NDDI-E) was employed for the evaluation of depression [17]. We administered the Spanish validated version [18]. The scores were gathered following the experiences in the preceding two weeks. In the Spanish version, scores higher than 13 were considered to suffer from major depressive episodes. Previous diagnosis of depression was obtained from the medical history. The diagnosis of anxiety and depression was performed by a psychiatrist or a neurologist based on an assessment by semi-structured interview.

The Epworth Sleepiness Scale (ESS) measures a subject's general time of daytime sleepiness [19]. The participants answered the Spanish validated version [20]. The Spanish version was considered equivalent to the original version, with scores higher than 10 reflecting sleepiness. In addition to the ESS, we collected the information about the number of sleeping hours, any sleep disturbance, 
waking up during the night, and problems with falling asleep. These last three sleep conditions were considered as binary variables. For example, if a patient woke up regularly during the night in the follow-up period, the value of the corresponding variable was taken as "yes" ("no" otherwise). Diagnosis of SAHS was obtained from the medical history based on prior evaluation performed by a sleep medicine specialist, which includes a polysomnography evaluation.

The aforementioned questionnaires were fulfilled during follow-up visits, and each diagnosis different to epilepsy treated in this study (depression, anxiety, and SAHS) was previous with respect to these follow-up visits. As secondary variables of interest, the available specific type of seizures and epilepsy according to the International League Against Epilepsy classification of epilepsies (2017) [21] and type of AED were gathered (see the supplementary tables).

\section{Statistical analysis}

Continuous data were reported using the mean and standard deviation and the median and interquartile range (difference between the third and first quartiles). Group proportions were obtained for the categorical variables.

To analyze the relationship between previous diagnosis of anxiety, depression, and SAHS and the scores of GAD7, NDDI-E, and ESS, respectively, we designed a logistic regression model. The objective of this model was to check the suitability of the corresponding tools in real clinical practice. In each model, the dependent variable was the diagnosis, and the independent variable was the score from the pertinent scale, considering the original numerical score and the categorical score obtained from the commented cut-offs in separate analyses. Results of logistic regression models are reported with the odds ratio (OR) and the 95\% confidence interval. As secondary analysis, we evaluated the association of seizure frequency and the diagnosis of anxiety and depression with the specific types of epilepsy and AED. In this analysis, we considered exclusively those types and drugs used by at least $20 \%$ of the patients.

For the assessment of the QoL with the QOLIE-31-P scores, generalized linear models (GLM) with a Gaussian distribution were employed. A stepwise backward model was implemented to obtain the final multivariate model, including in the initial entry the GAD-7, NDDI-E, and ESS numerical scores, the demographic and clinical variables, the sleep disorders variables, and the variables related to the types of epilepsy and AED mentioned in the previous paragraph. We aimed at building an explanatory model, i.e., establishing the relationship between the independent variables of study and the QoL, rather than obtaining the best predictive model. GLM results are reported with the regression coefficient and the $95 \%$ confidence interval. The analysis was conducted for the total score and for the scores from seven subscales (all except the health state item). The final results for each multivariate model were corrected for multiple comparisons using the Benjamini-Hochberg false discovery rate procedure. In an additional secondary analysis, we assessed specifically the relationship between the QOLIE-31-P scores, considering each subscale and the global score, and the presence of at least one epileptic seizure per month (binary variable).

An alpha value of 0.05 was set as the threshold for statistical significance. Data were analyzed using R statistical software, version 3.5.2. In case of missing values in any of the QOLIE-31-P questions, the guidelines of the original reference were followed [14]. Complete case analysis was performed in case of missing data in the other variables.

\section{Results}

A total of 85 patients were recruited. Among them, 49 patients answered the QOLIE-31-P. Forty-eight patients were included in the final sample for the analysis of the QoL, while one subject was excluded due to excessive amounts of missing data. The lack of answers of the QOLIE-31-P questionnaire was produced because it is considerably longer than the other assessment tools and more restrictive because patients are not allowed to answer it in case of seizure in the last $4 \mathrm{~h}$ (or generalized clonic seizure in the last $24 \mathrm{~h}$ ). Description of the diverse main demographic and clinical variables included in the study is shown in Table 1. The overall distribution of AED with the most frequent and the older generation treatments can be found in Supplementary Tables 1 and 2.

\section{Analysis of GAD-7, NDDI-E, ESS, and clinical variables}

The previous diagnosis of anxiety was significantly associated with the GAD-7 categorical $(O R=14.5[2.1,295.5]$, $p=0.020)$ and numerical scores $(O R=1.21[1.09,1.36]$, $p<0.001)$. The diagnosis of depression was significantly associated with the NDDI-E categorical $(O R=6.3[1.2$, 48.0], $p=0.043)$ and numerical scores $(O R=1.24$ [1.08, 1.42 ],$p=0.002$ ). The number of patients with diagnosis of anxiety and depression according to the GAD-7 and NDDIE scores, respectively, is shown in Table 2, together with the description of the distribution of GAD-7 and NDDI-E scores.

Regarding the relationship between the diagnosis of anxiety and depression and the types of epilepsy, no significant associations were found with either generalized epilepsy $(10 / 85=11.8 \%)$ or tonic-clonic seizures $(9 / 85=10.6 \%)$. The diagnosis of depression presented 
Table 1 Characteristics of the patients with epilepsy

\begin{tabular}{llll}
\hline Characteristics & Number $(\%)$ & Mean (SD) & Median [IQR] \\
\hline $\begin{array}{l}\text { Age (years, } N=85) \\
\text { Sex }\end{array}$ & $44.3(17.4)$ & $41[25]$ \\
$\quad$ Male & $44(51.8)$ & & \\
$\quad$ Female & $41(48.2)$ & & \\
Duration of epilepsy (years, $N=85)$ & & $21.5(15.9)$ & $18[24]$ \\
Seizure frequency (attacks/month, $N=84)$ & & $1.7(4.9)$ & $0[1]$ \\
Non-epileptic paroxysmal events $(N=85)$ & $6(7.1)$ & \\
Number of antiepileptic drugs $(N=85)$ & & & \\
Number of drugs $\geq 2$ & $48(56.5)$ & \\
Diagnosis of anxiety $(N=85)$ & $14(16.5)$ & & \\
Diagnosis of depression $(N=85)$ & $16(18.8)$ & & \\
Diagnosis of sleep apnea-hypopnea syndrome & $7(8.0)$ & & \\
$\quad(N=85)$ & & & \\
Number of sleeping hours $(N=73)$ & & & \\
Sleep disturbance $(N=84)$ & $32(38.1)$ & & \\
Waking up during the night $(N=84)$ & $28(33.3)$ & & \\
Problems to fall asleep $(N=84)$ & $24(28.6)$ & & \\
\hline
\end{tabular}

$S D$, standard deviation. $I Q R$, interquartile range
Table 2 Scale scores of anxiety (GAD-7), depression (NDDI-E), somnolence (ESS), and quality of life (QOLIE-31-P) of the patients with epilepsy

\begin{tabular}{llll}
\hline Scale scores & Number $(\%)$ & Mean $(\mathrm{SD})$ & Median [IQR] \\
\hline GAD-7 $(N=84)$ & & $7.6(5.4)$ & $6[7]$ \\
GAD-7 $>9$ & $22(28.5)$ & & \\
NDDI-E $(N=84)$ & & $12.4(4.4)$ & $11.5[5]$ \\
NDDI-E $>13$ & $28(33.3)$ & & \\
ESS $(N=77)$ & & $5.4(3.9)$ & $5[5]$ \\
ESS $>10$ & $11(14.3)$ & & \\
QOLIE-31-P $(N=48)$ & & $62.0(19.2)$ & $66.5[29.3]$ \\
Energy $(N=48)$ & & $54.0(25.9)$ & $60[31.3]$ \\
Mood $(N=47)$ & $60.6(25.2)$ & $64[38]$ \\
Daily activities $(N=47)$ & & $66.4(30.7)$ & $70[53.5]$ \\
Cognition $(N=48)$ & & $59.2(25.1)$ & $56.7[40.0]$ \\
Medication effects & & $40.5(30.4)$ & $33.3[44.4]$ \\
$\quad(N=48)$ & & \\
Seizure worry $(N=47)$ & & $51.8(27.7)$ & $49.3[49]$ \\
Overall quality of life & & & \\
$\quad(N=46)$ & & & \\
\hline
\end{tabular}

$S D$, standard deviation. $I Q R$, interquartile range

statistically significant association with focal epilepsy $(47 / 85=55.3 \% ; O R=4.5[1.3,20.7], p=0.029)$, particularly with focal impaired awareness seizures $(13 / 85=15.3 \% ;$ OR $=5.3[1.5,19.5], p=0.011)$. The diagnosis of anxiety was significantly related to temporal lobe epilepsy $(10 / 85=11.8 \% ; O R=4.3[1.0,18.1], p=0.044)$. No statistically significant association was found between the types of epilepsy and seizure frequency.
Concerning the analysis of specific AED, the previous diagnosis of depression was significantly associated with the use of lacosamide $(17 / 85=20 \% ; O R=4.6[1.4,15.4]$, $p=0.012)$. No significant associations were found with either between the previous diagnosis of anxiety and lacosamide or between the two most used drugs in our sample, which were lamotrigine $(22 / 85=25.9 \%)$ and levetiracetam $(27 / 85=31.8 \%)$, and anxiety or depression.

There was no statistically significant association between diagnosis of SAHS and ESS categorical $(p=0.993)$ and numerical scores $(p=0.658)$. The number of sleeping hours ( $p=0.563$ for categorical ESS, $p=0.288$ for numerical ESS), sleep disturbance ( $p=0.634$ for categorical ESS, $p=0.937$ for numerical ESS), waking up during the night ( $p=0.501$ for categorical ESS, $p=0.733$ for numerical ESS), and problems to fall asleep ( $p=0.539$ for categorical ESS, $p=0.859$ for numerical ESS) were not significantly associated with ESS. The distribution of the ESS scores and the number of patients with sleepiness according to the corresponding scores are shown in Table 2.

\section{Univariate analysis of QOLIE-31-P}

A statistically significant negative association was found between NDDI-E and the QOLIE-31-P total score and the QOLIE-31-P score from every subscale. The same results were found with GAD-7, except for the medication effects subscale. No significant associations were identified with the ESS.

QOLIE-31-P total and energy scores were significantly associated (negative association) with sleep disturbance, 
waking up during the night, and problems falling asleep. QOLIE-31-P mood and overall QoL scores were significantly associated (negative association) with sleep disturbance and problems to fall asleep. QOLIE-31-P daily activity score was significantly associated (negative association) with problems to fall asleep. Lower QOLIE-31-P seizure worry scores (higher worry) were significantly associated with waking up during the night.

Statistically significant lower scores (worse situation) were identified for female sex in QOLIE-31-P total, mood, daily activities, seizure worry, and overall QoL. Statistically significant positive relationship was observed between age and QOLIE-31-P medication effect score (better or higher scores with higher age). Drug-resistant epilepsy and the number of AED presented statistically significant lower QOLIE-31-P total, mood, cognition (only drug-resistant epilepsy), and medication effect scores (worse scores for every case). Regarding the diverse types of epilepsy, temporal lobe epilepsy was associated with lower QOLIE-31-P total and seizure worry scores (worse scores for both scales).

With respect to the assessment of the QOLIE-31-P scores and the presence of at least one epileptic seizure per month, lower overall QoL subscale scores were associated with the presence of monthly epileptic seizures $(\beta=-11.1$, $p=0.049$ ). No significant associations were found for the remaining subscales or the final QOLIE-31-P score.

Results from the univariate models are shown in Fig. 1 and Supplementary Tables 3-10. The description of the distribution of each QOLIE-31-P score is shown in Table 2.

\section{Multivariate analysis of QOLIE-31-P}

The final model included GAD-7 score, NDDI-E score, the number of sleeping hours, problems to fall asleep, drugresistant epilepsy, and sex as covariates. Four of these variables showed statistically significant negative association after correction for multiple comparisons. These variables were the GAD-7 numerical score $(\beta=-1.21$, adjusted $p=0.006)$, the NDDI-E numerical score $(\beta=-1.42$, adjusted $p=0.006)$, drug-resistant epilepsy $(\beta=-8.08$, adjusted $p=0.045)$, and female sex $(\beta=-7.83$, adjusted $p=0.034)$. The interpretation of the GAD-7 and NDDI-E results is that, for each additional point, the QOLIE-31-P total score is 1.21 and 1.42 points lower (worse QoL), respectively. The interpretation of the other two coefficients is that patients with drug-resistant epilepsy and women present 8.08 and 7.83 less QOLIE-31-P points than patients with less than two AED and men. The complete results are shown in Table 3.

With respect to the QOLIE-31-P subscale scores, GAD-7 was significantly associated with lower or worse energy $(\beta=-1.96$, adjusted $p=0.006), \operatorname{mood}(\beta=-3.27$, adjusted $p<0.001)$, daily activities $(\beta=-1.93$, adjusted $p=0.020)$, and seizure worry $(\beta=-2.54$, adjusted $p<0.001)$ scores. NDDI-E showed a statistically significant association with lower energy $(\beta=-1.97$, adjusted $p=0.025)$, daily activities $(\beta=-2.61$, adjusted $p=0.018)$, medication effects $(\beta=-1.79$, adjusted $p=0.038)$, and overall QoL $(\beta=-1.89$, adjusted $p<0.001)$ scores, which reflect worse situation in relation to higher levels of depression in each scale. Sleep disturbance was significantly associated with lower energy $(\beta=-14.77$, adjusted $p=0.025)$ and $\operatorname{mood}(\beta=-12.40$, adjusted $p=0.027)$ scores. Lower QOLIE-31-P scores (worse QoL) were also found in the following associations: seizure frequency and mood score $(\beta=-2.46$, adjusted $p=0.023)$, drug-resistant epilepsy and medication effects score $(\beta=-16.93$, adjusted $p=0.038$ ), problems to fall asleep and overall QoL score $(\beta=-11.64$, adjusted $p=0.022)$, female sex and seizure worry $(\beta=-21.29$, adjusted $p=0.005)$, and overall QoL ( $\beta=-8.52$, adjusted $p=0.049$ ) scores. In contrast, statistically significant associations with higher scores (better QoL) were found between age and medication effects score $(\beta=0.61$, adjusted $p=0.024)$ and between duration of epilepsy history and energy score $(\beta=0.41$, adjusted $p=0.041)$. Generalized epilepsy was associated with lower daily activity score $(\beta=-20.31$, adjusted $p=0.041)$, while focal epilepsy was related to higher energy score $(\beta=13.23$, adjusted $p=0.028)$. The use of lacosamide was associated with higher energy score $(\beta=16.62$, adjusted $p=0.036)$. Regarding the QOLIE-31-P cognition score, no model with multiple covariates presented significant results.

The significance for the QOLIE-31-P total and the subscale scores are shown in Fig. 1. The results from the models adjusted for multiple covariates, except the QOLIE-31-P total and cognition scores, are shown in Supplementary Tables 11-16.

\section{Discussion}

The present study provides a complete perspective of the QoL in patients with epilepsy in relationship with anxiety, depression, and sleep based on the analysis of quantitative scales specifically designed for patients with epilepsy. The individual assessments have been carried out in the literature but not a simultaneous analysis in real life, especially considering sleep. In this study, a relationship between worse QoL and higher scores from tools widely used to screen depression and anxiety in patients with epilepsy, NDDI-E and GAD-7, was observed. Furthermore, an association between worse QoL and sleep disturbance was found, although no tool was related to previous diagnosis of either insomnia or SAHS. Other factors linked to worse QoL in patients with epilepsy were drug-resistant epilepsy and female sex. 
Fig. 1 Relationship between quality of life (QOLIE-31-P) and other variables. A Anxiety (GAD-7) and depression (NDDI-E). B Sleep variables. C Other variables

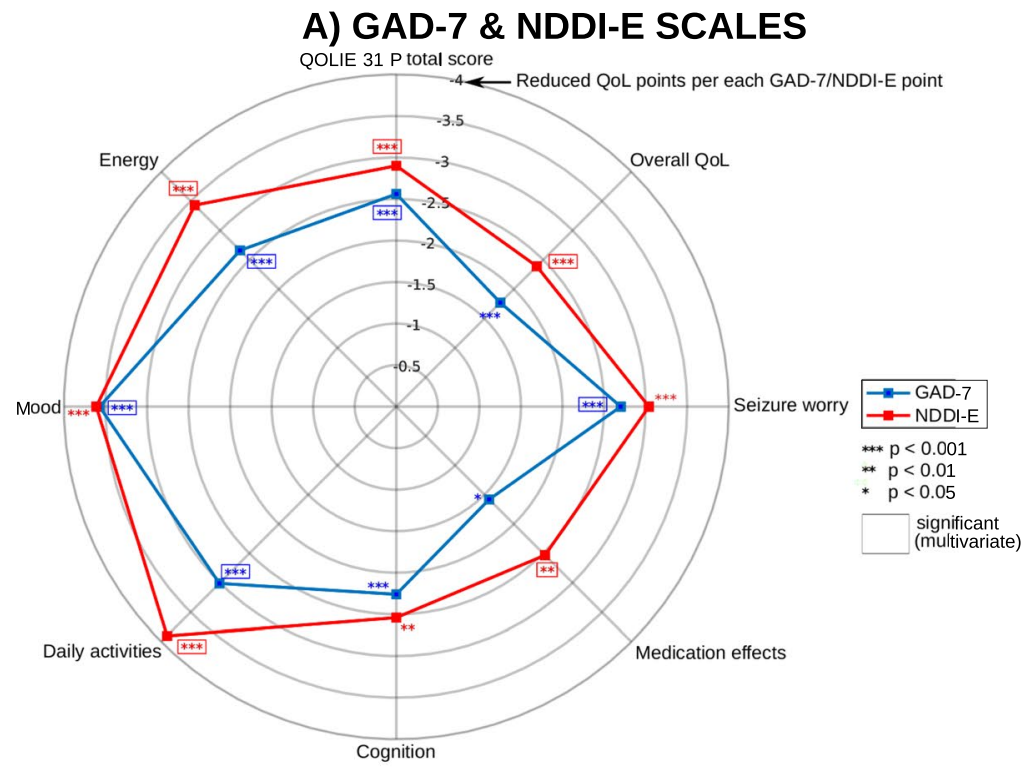

B) SLEEP VARIABLES

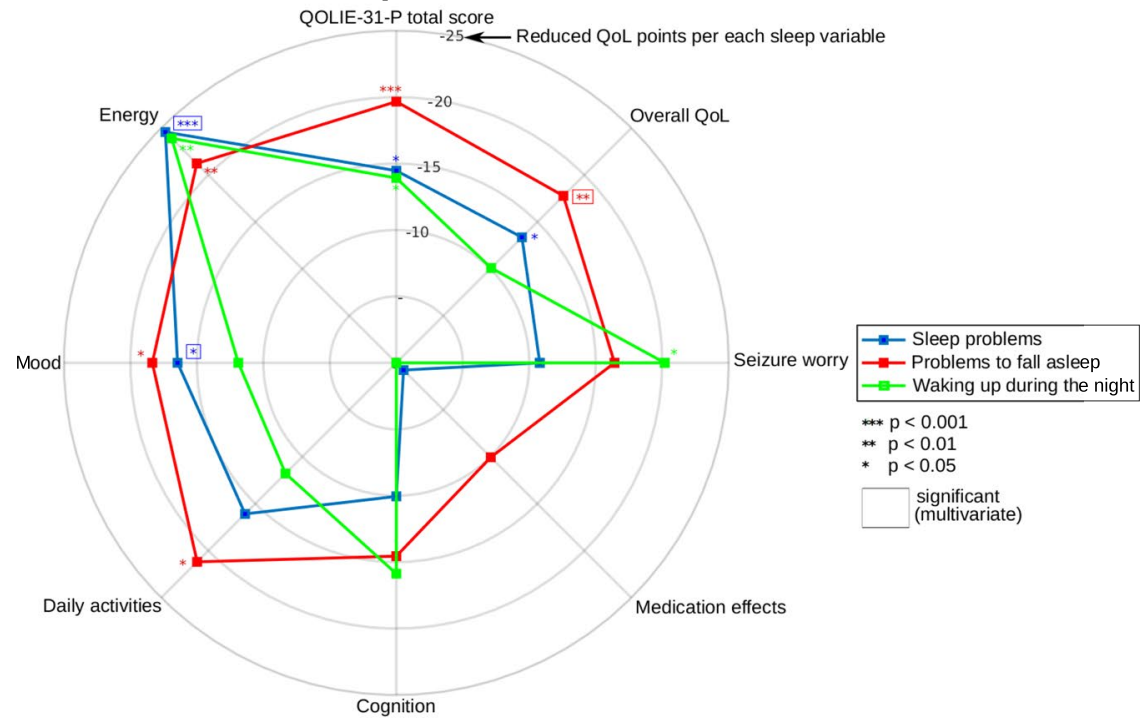

C) OTHER CATEGORICAL VARIABLES

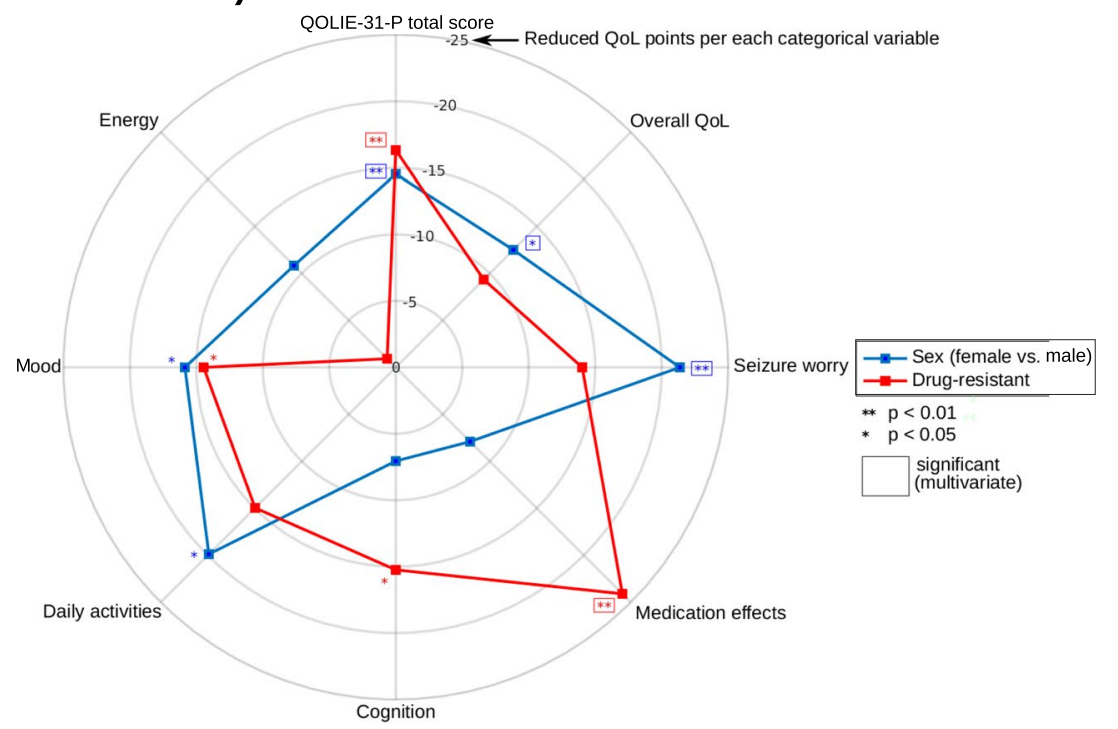


Table 3 QOLIE-31-P total score model adjusted for multiple covariates

\begin{tabular}{llll}
\hline Characteristics & Coefficient [95\% CI] & $p$ value & $\begin{array}{l}\text { Adjusted } \\
p \text { value } \\
\text { (FDR) }\end{array}$ \\
\hline Independent term & $98.19[81.17,115.22]$ & $<0.001$ & $<0.001$ \\
GAD-7 & $-1.21[-1.91,-0.51]$ & 0.002 & 0.006 \\
NDDI-E & $-1.42[-2.27,-0.57]$ & 0.002 & 0.006 \\
Number of sleeping & $0.27[-1.67,2.20]$ & 0.79 & 0.79 \\
$\quad$ hours & & & \\
Problems to fall asleep & -8.50 & 0.049 & 0.057 \\
& {$[-16.68,-0.32]$} & & \\
Drug-resistant epilepsy & -8.08 & 0.032 & 0.045 \\
& {$[-15.16,-1.00]$} & & \\
Sex (female vs. male) & -7.83 & 0.019 & 0.034 \\
& {$[-14.08,-1.58]$} & & \\
\hline
\end{tabular}

The associations between GAD-7 or NDDI-E and worse QoL scores are in line with the results reported by Micoulaud-Franchi et al. [22]. In the aforementioned study, the authors reported a negative correlation between GAD-7 and QOLIE-31 scores and between NDDI-E and QOLIE-31 scores, similar results compared to our association results. In the same study, the authors also identified negative correlation between QOLIE-31 and the adverse event profile questionnaire, a tool employed to assess the effects of AED. It is worth noting that, in our study, GAD-7 and NDDI-E scores were not only related to the total score of QOLIE31-P, but also related to the scores from the diverse QOLIE31-P subscales. Moreover, recent studies have shown that depressive symptoms and emotional dysregulation are linked by a bidirectional relationship and are significantly associated with worse quality of life and higher stigmatization feelings [23, 24].

Regarding the effect of AED, in our study, drug-resistant epilepsy was associated with worse QoL, as demonstrated in previous studies[25, 26]. In our study, despite being statistically significant only in the medication effect QOLIE-31-P subscale model, drug-resistant epilepsy was shown as a significant predictor of worse QoL. This result may reflect the importance of adverse effects of AED, especially in patients with drug-resistant epilepsy, who use a higher number and combinations of AED. Interestingly, older patients seemed to present a better QoL with respect to the medication effects according to the QOLIE-31-P results from our study. Previous studies have reported that seniors with epilepsy do not have poorer QoL compared with the general epilepsy populations $[6,27]$. Our results could be partially explained by the particularly careful selection of the anticonvulsant drugs in this particular group of people in an epilepsy unit, which may reduce secondary adverse effects. AED choice needs a careful evaluation in elderly due to the susceptibility to adverse effects because of the physiologic changes and the frequent comorbidities [28].

In addition to the GAD-7 and NDDI-E results, we have identified the associations between diverse self-reported sleep disorders and lower QoL scores, especially regarding aspects related to energy, mood, and overall QoL. In line with our study, especially with regard to our univariate analysis, sleep disturbances have been associated with lower QoL in patients with epilepsy and controls, with more pronounced effect on the patients [4].

Moreover, we found a relationship between depression and focal epilepsy. Previous series have reported that depression is associated with temporal lobe epilepsy related to mesial temporal sclerosis [29]. Besides, we found a statistically significant association between anxiety and temporal lobe epilepsy, in line with previous reports of interictal mood and personality disorders in temporal lobe epilepsy [30]. Moreover, we found lower scores in QOLIE-31-P in patients with temporal lobe epilepsy in our study, which could be explained by the presence of anxiety comorbidity in patients [31], independently of the seizure frequency according to our results. These patients presented lower QoL scores in the univariate model but not in the model with multiple covariates. Taking into account the relationships between depression and temporal lobe epilepsy and worse QoL and GAD-7 and NDDI-E scores, these patients may present lower QoL because of depression but not due to specific temporal lobe epilepsy features.

Regarding the relationship between previous diagnosis of anxiety and depression disorders and specific AED, we found an association between the use of lacosamide and depression, which might be explained by the neutral or positive mood effect of lacosamide [32] which can influence clinicians' selection of this drug in patients with depression [33]. Interestingly, we found that higher scores of the QOLIE-31-P energy subscale were associated with the use of lacosamide. This may be related to the possible positive or mood stabilizer effect of lacosamide on patients with symptoms of depression [34].

In contrast to symptoms of depression and anxiety, we were unable to associate a specific scale, the ESS, with either sleep disorders or previous diagnosis of SAHS. In accordance with our results, in previous studies, no significant ESS differences have been identified in patients with epilepsy with sleep disorders and excessive daytime sleepiness compared to controls [4]. However, a meta-analysis has demonstrated that the prevalence of SAHS in people with epilepsy is higher than in the general population [12]. The absence of an association between ESS and SAHS could be explained by the adequate use of continuous positive airway pressure (CPAP) in these patients [35], considering also the low number of patients with SAHS in our sample. In addition, excessive daytime sleepiness could be related not only 
to the presence of SAHS, but also to adverse effects of drugs [11].

In this study, we found lower QOLIE-31-P total scores in female compared to male patients with epilepsy and also worse overall QoL and seizure worry scores (lower values in both cases) after adjusting for multiple covariates. In contrast to our study, no statistically significant QOLIE-31-P differences between men and women were found in a study conducted in Poland with a similar sample size of patients with epilepsy [36]. It is worth noting that all the patients from the Polish study suffered from drug-resistant epilepsy, while in our study, approximately half of patients suffered from the same condition. Moreover, considering that approximately a third of women with epilepsy seizure frequency worsens around menstruation (catamenial epilepsy) [37], this last factor may explain the statistically significant lower (worse) QOLIE-31-P scores related to seizure worry in women. Another possible contributor to the worse perceived QoL in women could be the important amount of women of childbirth age, which has been previously associated with worse QoL [38]. Furthermore, differences in QoL regarding sex distribution have been observed in other neurological chronic diseases such as Parkinson disease, with women presenting worse physical functioning and socioemotional QoL and men worse cognition QoL [39].

Interestingly, seizure frequency was significantly associated only with QOLIE-31-P mood score, with no significant influence on other QoL variables. In our sample, it must be considered that most patients presented a relative low seizure frequency, which may explain this result. Nevertheless, in other studies such as that conducted by Siarava et al., using a different QoL tool, the BREF-World Health Organization Quality of Life questionnaire, no significant effect of seizure frequency on the diverse subscales was reported [40].

This study has some limitations. Firstly, the sample size might be small. The main reason is that the recruitment was interrupted because of the lockdown caused by the COVID-19 lockdown in countries like Spain, which might have caused changes in the QoL of patients with epilepsy which was completed by only $58 \%$ of the sample. Anyway, the sample size was big enough to obtain significant associations. Secondly, considering that the patients were recruited from a specialized epilepsy unit, there could be a potential bias related to the inclusion of patients with more severe epilepsy. However, more than half of the patients from our sample presented a relative low seizure frequency, between one and 11 attacks per year (less than one attack per month), reflecting a non-severe status. Additionally, the sample might not be completely representative of all patients with epilepsy due to the recruitment from a refractory epilepsy unit, although the close follow-up of the patients by specialized neurologists reduced uncertainty related to the diagnosis and variables such as seizure frequency. With regard to the lack of QOLIE-31-P answers, cognition bias may have been present because some questions are more difficult to understand with respect to the questions from other tools.

\section{Conclusions}

Our data show that anxiety and depression are associated with the QoL in real clinical practice in patients with epilepsy. We found an association between depression and focal epilepsy and between anxiety and temporal lobe epilepsy. Moreover, female sex and drug-resistant epilepsy showed the highest impact on QoL in this population. In addition, sleep disturbances seem to be associated with particular aspects of the QoL. Interestingly, older age was not associated with a worse QoL. Therefore, the evaluation of mood and anxiety disturbances through the aforementioned scales, as well as sleep disturbances, in patients with epilepsy in real-life clinical practice is important in order to adequately diagnose and manage comorbidities and to improve QoL of these patients. Regarding the use of the specific tools, it may be helpful to identify patients with current depression or anxiety. Screening of mood disorders and QoL seems especially important in patients with temporal lobe epilepsy. Further studies based on a longitudinal follow-up would be necessary to evaluate the effect of AED.

Supplementary Information The online version contains supplementary material available at https://doi.org/10.1007/s10072-021-05595-3.

Funding ÁP-G was supported by Junta de Castilla y León (Spain) and the European Social Fund (ID: 376062, Base de Datos Nacional de Subvenciones).

Data availability The data used in this study are available upon reasonable request to the corresponding author.

Code availability The $\mathrm{R}$ code used for statistical analysis is available upon reasonable request to the corresponding author.

\section{Declarations}

Ethics approval The study was approved by the Ethics Committee of the Hospital Universitario de la Princesa (Madrid, Spain).

Consent to participate A written informed consent was signed by the patients who agreed to participate in the study.

Conflict of interest The authors declare no competing interests.

\section{References}

1. Bell GS, Sander JW (2001) The epidemiology of epilepsy: the size of the problem. Seizure 10:306-316. https://doi.org/10.1053/seiz. 2001.0584 
2. Schachter SC (2006) Quality of life for patients with epilepsy is determined by more than seizure control: the role of psychosocial factors. Expert Rev Neurother 6:111-118. https://doi.org/10.1586/ 14737175.6.1.111

3. Schipper H (1983) Why measure quality of life? Can Med Assoc J 128:1367-1370

4. Gutter T, Callenbach PMC, Brouwer OF, de Weerd AW (2019) Prevalence of sleep disturbances in people with epilepsy and the impact on quality of life: a survey in secondary care. Seizure 69:298-303. https://doi.org/10.1016/j.seizure.2019.04.019

5. Josephson CB, Jetté N (2017) Psychiatric comorbidities in epilepsy. Int Rev Psychiatry 29:409-424. https://doi.org/10.1080/ 09540261.2017.1302412

6. Baranowski CJ (2018) The quality of life of older adults with epilepsy: a systematic review. Seizure Eur J Epilepsy 60:190-197. https://doi.org/10.1016/j.seizure.2018.06.002

7. Scott AJ, Sharpe L, Hunt C, Gandy M (2017) Anxiety and depressive disorders in people with epilepsy: a meta-analysis. Epilepsia 58:973-982. https://doi.org/10.1111/epi.13769

8. de Araújo Filho GM, Martins DP, Lopes AM, de Jesus BB, Furlan AER, Oliveira CIF et al (2018) Oxidative stress in patients with refractory temporal lobe epilepsy and mesial temporal sclerosis: possible association with major depressive disorder? Epilepsy Behav 80:191-196. https://doi.org/10.1016/j.yebeh.2017.12.025

9. Munger Clary HM, Snively BM, Hamberger MJ (2018) Anxiety is common and independently associated with clinical features of epilepsy. Epilepsy Behav 85:64-71. https://doi.org/10.1016/j. yebeh.2018.05.024

10. Kataria L, Vaughn BV (2016) Sleep and epilepsy. Sleep Med Clin 11:25-38. https://doi.org/10.1016/j.jsmc.2015.10.008

11. Çilliler AE, Güven B (2020) Sleep quality and related clinical features in patients with epilepsy: a preliminary report. Epilepsy Behav 102. https://doi.org/10.1016/j.yebeh.2019.106661

12. Lin Z, Si Q, Xiaoyi Z (2017) Obstructive sleep apnoea in patients with epilepsy: a meta-analysis. Sleep Breath 21:263-270. https:// doi.org/10.1007/s11325-016-1391-3

13. Kwan P, Arzimanoglou A, Berg AT, Brodie MJ, Allen Hauser W, Mathern G et al (2010) Definition of drug resistant epilepsy: consensus proposal by the ad hoc task force of the ILAE Commission on Therapeutic Strategies. Epilepsia 51:1069-1077. https:// doi.org/10.1111/j.1528-1167.2009.02397.x

14. Cramer JA, Van Hammée G (2003) Maintenance of improvement in health-related quality of life during long-term treatment with levetiracetam. Epilepsy Behav 4:118-123. https://doi.org/10.1016/ s1525-5050(03)00004-0

15. Spitzer RL, Kroenke K, Williams JBW, Löwe B (2006) A brief measure for assessing generalized anxiety disorder: the GAD-7. Arch Intern Med 166:1092-1097. https://doi.org/10.1001/archi nte.166.10.1092

16. García-Campayo J, Zamorano E, Ruiz MA, Pardo A, PérezPáramo M, López-Gómez V et al (2010) Cultural adaptation into Spanish of the generalized anxiety disorder-7 (GAD-7) scale as a screening tool. Heal Qual Life Outcomes 8:8. https://doi.org/10. 1186/1477-7525-8-8

17. Friedman DE, Kung DH, Laowattana S, Kass JS, Hrachovy RA, Levin HS (2009) Identifying depression in epilepsy in a busy clinical setting is enhanced with systematic screening. Seizure 18:429-433. https://doi.org/10.1016/j.seizure.2009.03.001

18. Di Capua D, Garcia-Garcia ME, Reig-Ferrer A, Fuentes-Ferrer M, Toledano R, Gil-Nagel A et al (2012) Validation of the Spanish version of the Neurological Disorders Depression Inventory for Epilepsy (NDDI-E). Epilepsy Behav 24:493-496. https://doi.org/ 10.1016/j.yebeh.2012.06.005

19. Johns MW (1991) A new method for measuring daytime sleepiness: the Epworth Sleepiness Scale. Sleep 14:540-545. https:// doi.org/10.1093/sleep/14.6.540
20. Chiner E, Arriero JM, Signes-Costa J, Marco J, Fuentes I (1999) Validation of the Spanish version of the Epworth Sleepiness Scale in patients with sleep apnea syndrome. Arch Bronconeumol 35:422-427. https://doi.org/10.1016/S0300-2896(15)30037-5

21. Scheffer IE, Berkovic S, Capovilla G, Connolly MB, French J, Guilhoto L, Hirsch E, Jain S, Mathern GW, Moshé SL, Nordli DR, Perucca E, Tomson T, Wiebe S, Zhang YH, Zuberi SM (2017) ILAE classification of the epilepsies: position paper of the ILAE Commission for Classification and Terminology. Epilepsia 58(4):512-521. https://doi.org/10.1111/epi.13709

22. Micoulaud-Franchi J-A, Bartolomei F, Duncan R, McGonigal A (2017) Evaluating quality of life in epilepsy: the role of screening for adverse drug effects, depression, and anxiety. Epilepsy Behav 75:18-24. https://doi.org/10.1016/j.yebeh.2017.07.016

23. Tombini M, Assenza G, Quintiliani L, Ricci L, Lanzone J, Ulivi M, Di Lazzaro V (2020) Depressive symptoms and difficulties in emotion regulation in adult patients with epilepsy: association with quality of life and stigma. Epilepsy Behav 107:107073. https://doi.org/10.1016/j.yebeh.2020.107073

24. Tombini M, Assenza G, Quintiliani L, Ricci L, Lanzone J, De Mojà R, Ulivi M, Di Lazzaro V (2019) Epilepsy-associated stigma from the perspective of people with epilepsy and the community in Italy. Epilepsy Behav 98(Pt A):66-72. https://doi.org/10.1016/j. yebeh.2019.06.026

25. Baker GA, Jacoby A, Buck D, Stalgis C, Monnet D (1997) Quality of life of people with epilepsy: a European study. Epilepsia 38:353-362. https://doi.org/10.1111/j.1528-1157.1997.tb01128.x

26. Sander JW, Shorvon SD (1996) Epidemiology of the epilepsies. J Neurol Neurosurg Psychiatry 61:433-443. https://doi.org/10. 1136/jnnp.61.5.433

27. Laccheo I, Ablah E, Heinrichs R, Sadler T, Baade L, Liow K (2008) Assessment of quality of life among the elderly with epilepsy. Epilepsy Behav 12:257-261. https://doi.org/10.1016/j. yebeh.2007.09.003

28. Kirmani BF, Robinson DM, Kikam A, Fonkem E, Cruz D (2014) Selection of antiepileptic drugs in older people. Curr Treat Options Neurol 16:295. https://doi.org/10.1007/s11940-014-0295-4

29. Quiske A, Helmstaedter C, Lux S, Elger CE (2000) Depression in patients with temporal lobe epilepsy is related to mesial temporal sclerosis. Epilepsy Res 39(2):121-125. https://doi.org/10.1016/ s0920-1211(99)00117-5

30. Perini GI, Tosin C, Carraro C, Bernasconi G, Canevini MP, Canger R, Pellegrini A, Testa G (1996) Interictal mood and personality disorders in temporal lobe epilepsy and juvenile myoclonic epilepsy. J Neurol Neurosurg Psychiatry 61(6):601-605. https://doi.org/10.1136/jnnp.61.6.601

31. Chen YY, Huang S, Wu WY, Liu CR, Yang XY, Zhao HT, Wu LC, Tan LZ, Long LL, Xiao B (2018) Associated and predictive factors of quality of life in patients with temporal lobe epilepsy. Epilepsy Behav 86:85-90. https://doi.org/10.1016/j.yebeh.2018. 06.025

32. Rocamora R, Ley M, Molins A, Toledo M, Sansa G, Bertol V, Becerra JL, Carreño M, Mauri JÁ (2018) Effect of lacosamide on depression and anxiety symptoms in patients with focal refractory epilepsy: a prospective multicenter study. Epilepsy Behav 79:87-92. https://doi.org/10.1016/j.yebeh.2017.10.032

33. Moseley BD, Cole D, Iwuora O, Strawn JR, Privitera M (2015) The effects of lacosamide on depression and anxiety in patients with epilepsy. Epilepsy Res 110:115-118. https://doi.org/10. 1016/j.eplepsyres.2014.12.007

34. Alfaro A, Asensio M, García-Escrivá A, Medrano V, Salom JM, Tortosa D, Palao S, Lezcano M, Berenguer L, Navarro M, Cerdán M, Buendía JF, Giner JC (2019) LAM study: effects of lacosamide on behaviour and quality of life in patients with epilepsy. Neurologia 34(1):1-6. English, Spanish. https://doi.org/10.1016/j.nrl. 2016.10.007 
35. Zimmermann C, Köhler D, Schönhofer B (2000) Wertigkeit der retrospektiven Einschätzung der Epworth-Schläfrigkeitsskala nach länger dauernder CPAP-Therapie bei obstruktiver, schlafbezogener Atmungsstörung [Value of retrospective assessment of the Epworth Sleepiness Scale after long-term CPAP therapy. Pneumologie 54:572-4. https://doi.org/10.1055/s-2000-9193

36 Bala A, Szantroch M, Gleinert A, Rysz A, Marchel A (2016) Differences in quality of life of women and men with drug-resistant epilepsy in Poland. Epilepsy Behav 60:94-8. https://doi.org/10. 1016/j.yebeh.2016.04.041

37 Stephen LJ, Harden C, Tomson T, Brodie MJ (2019) Management of epilepsy in women. Lancet Neurol 18:481-91. https://doi.org/ 10.1016/S1474-4422(18)30495-2

38 Santos AMC, Castro-Lima H, Matos MAA, Brito MB (2018) Quality of life among women with epilepsy during their reproductive years. Epilepsy Behav 85:10-3. https://doi.org/10.1016/j. yebeh.2018.04.028
39 Ophey A, Eggers C, Dano R, Timmermann L, Kalbe E (2018) Health-related quality of life subdomains in patients with Parkinson's disease: the role of gender. Parkinsons Dis 1(2018):6532320. https://doi.org/10.1155/2018/6532320

40. Siarava E, Hyphantis T, Katsanos AH, Pelidou S-H, Kyritsis AP, Markoula S (2019) Depression and quality of life in patients with epilepsy in Northwest Greece. Seizure 66:93-98. https://doi.org/ 10.1016/j.seizure.2019.02.012

Publisher's note Springer Nature remains neutral with regard to jurisdictional claims in published maps and institutional affiliations. 